\title{
MULTIPLIER HOPF ALGEBRAS
}

\author{
A. VAN DAELE
}

\begin{abstract}
In this paper we generalize the notion of Hopf algebra. We consider an algebra $A$, with or without identity, and a homomorphism $\Delta$ from $A$ to the multiplier algebra $M(A \otimes A)$ of $A \otimes A$. We impose certain conditions on $\Delta$ (such as coassociativity). Then we call the pair $(A, \Delta)$ a multiplier Hopf algebra. The motivating example is the case where $A$ is the algebra of complex, finitely supported functions on a group $G$ and where $(\Delta f)(s, t)=f(s t)$ with $s, t \in G$ and $f \in A$. We prove the existence of a counit and an antipode. If $A$ has an identity, we have a usual Hopf algebra. We also consider the case where $A$ is a $*$-algebra. Then we show that (a large enough) subspace of the dual space can also be made into a $*$-algebra.
\end{abstract}

\section{INTRODUCTION}

If $G$ is a finite group and $A$ is the algebra of functions from $G$ to $\mathbb{C}$ with pointwise operations, then $A$ can be made into a Hopf algebra if we define comultiplication, counit, and antipode by

$$
(\Delta f)(s, t)=f(s t), \quad \varepsilon(f)=f(e), \quad(S f)(t)=f\left(t^{-1}\right),
$$

where $f \in A$ and $s, t \in G$ and where $e$ is the identity in $G$. We have $\Delta(A) \subseteq A \otimes A$ if we identify $A \otimes A$ with functions on $G \times G$.

This is no longer possible if $G$ is infinite. However, if then we take for $A$ the algebra of finitely supported complex functions and if we cut down one variable, we get functions in $A \otimes A$. Indeed, for all $f$ and $g$ in $A$ we have that $(\Delta f)(1 \otimes g)$ and $(\Delta f)(g \otimes 1)$ are in $A \otimes A$.

On the other hand, we have the following result. If $A$ is any Hopf algebra with comultiplication $\Delta$, then the maps $T_{1}, T_{2}: A \otimes A \rightarrow A \otimes A$, defined by

$$
T_{1}(a \otimes b)=\Delta(a)(1 \otimes b), \quad T_{2}(a \otimes b)=(a \otimes 1) \Delta(b)
$$

are bijections. Moreover, if $A$ is an algebra with identity and a comultiplication $\Delta$ such that the above maps are bijective, then $A$ is a Hopf algebra. (We give a proof of these results in $\S \S 2$ and 4.) This implies that not only $\Delta$ but also $S$ and $\varepsilon$ are determined by the two maps $T_{1}$ and $T_{2}$. And, in principle, one can translate the axioms in terms of these maps.

If then we drop the requirement that $A$ has an identity, we get a generalized notion of Hopf algebra such that also the above example, with an infinite group,

Received by the editors September 25, 1992.

1991 Mathematics Subject Classification. Primary 16W30; Secondary 17B37. 
fits into the scheme. We will introduce this notion in $\S 2$. We will use the terminology of multipliers (see appendix). And therefore we call these generalized Hopf algebras multiplier Hopf algebras.

In $\S 3$ we will prove the existence of a counit and in $\S 4$ the existence of an antipode. We formulate conditions to have that the antipode is a bijection. Such multiplier Hopf algebras are called regular. If $A$ is abelian, it is automatically regular. We also consider *-algebras. Also for these algebras, regularity is automatic. We consider these cases in $\S 5$.

If $A$ has an identity, we get a usual Hopf algebra. With our approach we see that the existence of (the unique) counit and antipode can be proved from certain necessary and sufficient conditions on the comultiplication $\Delta$.

In $\S 6$ we consider the dual space $A^{\prime}$. It is not possible to make $A^{\prime}$ into an algebra as for usual Hopf algebras. We have to take a suitable subspace $A^{*}$ of $A^{\prime}$. If $A$ is a *-algebra, then also $A^{*}$ is a $*$-algebra.

If $A$ is a finite-dimensional Hopf algebra, then $A^{\prime}$ is again a Hopf algebra. If $A$ is any Hopf algebra, this is no longer true because the obvious candidate for the comultiplication $\Delta$ on $A^{\prime}$ will not map into $A^{\prime} \otimes A^{\prime}$ but into a larger space. In many cases, one can find a large enough subalgebra $A^{0}$ of $A^{\prime}$ such that $\Delta\left(A^{0}\right) \subseteq A^{0} \otimes A^{0}$. The same problem arises for our generalized Hopf algebras. To solve this problem there is a need for a topological theory. Only in that case one can hope that the dual is again a Hopf algebra of the same kind. There are attempts in this direction (see, e.g., $[2,6]$ ) but they are not (yet) completely satisfactory.

We believe that this paper, although it is purely algebraic, contains ideas that could be helpful in developing the topological theory. In a recent paper [6], we also showed that multiplier Hopf algebras, as introduced here, provide a natural framework to study discrete quantum groups. They also give interesting, nontrivial examples of multiplier Hopf algebras.

For the theory of Hopf algebras we refer to the standard books of Abe [1] and Sweedler [3]. See also [4]. We will, also in the generalized case, always use $\Delta, \varepsilon, S$ for the comultiplication, the counit and the antipode. We will use $\Delta^{\prime}$ for the opposite comultiplication, i.e., $\Delta^{\prime}(a)=\sigma \Delta(a)$ where $\sigma$ is the flip from $A \otimes A$ to $A \otimes A$ defined by $\sigma(b \otimes c)=c \otimes b$.

We will only consider algebras $A$ with a nondegenerate product, i.e., if $a \in A$ and $a b=0$ for all $b \in A$, then $a=0$, and similarly if $b a=0$ for all $b \in A$, then $a=0$. Then we can consider the multiplier algebra $M(A)$ and $A$ will be a subalgebra of $M(A)$ (see appendix). Also we will only consider algebras over $\mathbb{C}$. By a $*$-algebra we mean an algebra with an involution $a \rightarrow a^{*}$ which is conjugate linear and an antihomomorphism.

We will consider tensor products of algebras and of linear maps on these algebras. We will identify $\mathbb{C} \otimes A$ and $A \otimes \mathbb{C}$ with $A$. We will use $l$ to denote the identity map. If, e.g., we have $f: A \rightarrow \mathbb{C}$ then $f \otimes l: A \otimes A \rightarrow A$ with $(f \otimes l)(a \otimes b)=f(a) b$. Finally, we will use $m$ to denote the multiplication as a linear map from $A \otimes A$ to $A$ defined by $m(a \otimes b)=a b$.

\section{DEFINITIONS AND EXAMPLES}

The following result is the motivation for our definitions in this section.

2.1 Proposition. If $A$ is a Hopf algebra, then the linear maps $T_{1}, T_{2}: A \otimes A \rightarrow$ $A \otimes A$, defined by 


$$
T_{1}(a \otimes b)=\Delta(a)(1 \otimes b), \quad T_{2}(a \otimes b)=(a \otimes 1) \Delta(b)
$$

are bijective.

Proof. Define linear maps $R_{1}, R_{2}: A \otimes A \rightarrow A \otimes A$ by

$$
\begin{aligned}
& R_{1}(a \otimes b)=((l \otimes S) \Delta(a))(1 \otimes b), \\
& R_{2}(a \otimes b)=(a \otimes 1)((S \otimes l) \Delta(b)) .
\end{aligned}
$$

By a straightforward application of the properties of $S$ and $\Delta$ one can show that $R_{1}$ is the inverse of $T_{1}$ and that $R_{2}$ is the inverse of $T_{2}$ : if we use the usual symbolic notations (see [1] and [3]), we get, e.g.,

$$
\begin{aligned}
\left(T_{1} R_{1}\right)(a \otimes b) & =T_{1} \sum_{(a)} a_{(1)} \otimes S\left(a_{(2)}\right) b=\sum_{(a)} a_{(1)} \otimes a_{(2)} S\left(a_{(3)}\right) b \\
& =\sum_{(a)} a_{(1)} \otimes \varepsilon\left(a_{(2)}\right) b=a \otimes b . \quad
\end{aligned}
$$

(Generalizations of these formulas are obtained in $\S 4$.)

If the antipode has an inverse, then also the other linear maps, defined by

$$
a \otimes b \rightarrow \Delta(a)(b \otimes 1), \quad a \otimes b \rightarrow(1 \otimes a) \Delta(b)
$$

are bijections. This follows, e.g., from the fact that $S^{-1}$ will be the antipode if we replace $\Delta$ by the opposite comultiplication $\Delta^{\prime}$.

We will show in the next sections that, if $A$ is an algebra over $\mathbb{C}$ with identity and a comultiplication such that the maps $T_{1}$ and $T_{2}$ are bijections, then $A$ is actually a Hopf algebra (see Theorem 4.7). This is the motivation for the notion of generalized Hopf algebras that we will introduce now.

So, in what follows, let $A$ be an algebra over $\mathbb{C}$, with a nondegenerate product. $A$ may or may not have an identity. We refer to the appendix for results on multipliers.

2.2 Definition. A comultiplication on $A$ is a homomorphism $\Delta: A \rightarrow$ $M(A \otimes A)$ such that

(i) $\Delta(a)(1 \otimes b) \in A \otimes A$ and $(a \otimes 1) \Delta(b) \in A \otimes A$ for all $a, b \in A$,

(ii) $\Delta$ is coassociative in the following sense:

$$
(a \otimes 1 \otimes 1)(\Delta \otimes l)(\Delta(b)(1 \otimes c))=(l \otimes \Delta)((a \otimes 1) \Delta(b))(1 \otimes 1 \otimes c)
$$

for all $a, b, c$ in $A$.

Remark that condition (i) makes sense because we have $A \otimes A \subseteq M(A) \otimes$ $M(A) \subseteq M(A \otimes A)$.

We now define our generalized Hopf algebras.

2.3 Definition. Let $A$ be an algebra over $\mathbb{C}$, with or without identity and let $\Delta$ be a comultiplication on $A$. We call $A$ a multiplier Hopf algebra if the linear maps $T_{1}, T_{2}: A \otimes A \rightarrow A \otimes A$, defined by

$$
T_{1}(a \otimes b)=\Delta(a)(1 \otimes b), \quad T_{2}(a \otimes b)=(a \otimes 1) \Delta(b)
$$

are bijective. We call $A$ regular if $\sigma \Delta$, where $\sigma$ is the flip, is again a comultiplication such that $(A, \sigma \Delta)$ is also a multiplier Hopf algebra. 
These conditions in fact imply that $\Delta$ is a nondegenerate homomorphism. Then by Proposition A.5 of the appendix, the homomorphisms $l \otimes \Delta$ and $\Delta \otimes l$ have unique extensions to $M(A \otimes A)$. Condition (ii) of Definition 2.2 then means nothing else but $(\Delta \otimes l) \Delta=(l \otimes \Delta) \Delta$. But we will always use coassociativity as formulated in Definition 2.2.

If $A$ has an identity this new notion of multiplier Hopf algebra coincides with the old one (see $\S 4$ ). In that case $\sigma \Delta$ is automatically a comultiplication. The algebra will be regular iff $S$ has an inverse. In general, if $A$ is abelian, regularity is automatic (see $\S 5$ ).

We are also interested in the case of a $*$-algebra.

2.4 Definition. If $A$ is a $*$-algebra, we call $\Delta$ a comultiplication if it is also a $*$-homomorphism. A multiplier Hopf $*$-algebra is a $*$-algebra with a comultiplication, making it into a multiplier Hopf algebra.

Also for a multiplier Hopf *-algebra, the regularity is automatic.

We now come back to our motivating example.

2.5 Example. Let $G$ be any group and let $A$ be the *algebra of complex, finitely supported functions on $G$. In this case $M(A)$ consists of all complex functions on $G$. Moreover $A \otimes A$ can be naturally identified with finitely supported complex functions on $G \times G$ so that $M(A \otimes A)$ is the space of all complex functions on $G \times G$. If we define $\Delta: A \rightarrow M(A \otimes A)$ by

$$
(\Delta f)(s, t)=f(s t)
$$

we will clearly get a $*$-homomorphism. If $f, g \in A$ then $(s, t) \rightarrow f(s t) g(t)$ and $(s, t) \rightarrow g(s) f(s t)$ will have finite support and so belong to $A \otimes A$. This gives condition (i) of Definition 2.2. The coassociativity condition (ii) is an immediate consequence of the associativity of the multiplication on $G$. So $\Delta$ is a comultiplication.

The map $T_{1}$ is bijective and its inverse $R_{1}$ is given by $\left(R_{1} f\right)(s, t)=$ $f\left(s t^{-1}, t\right)$ when $f \in A \otimes A$ and $s, t \in G$. Similarly, the map $T_{2}$ is bijective and its inverse $R_{2}$ is given by $\left(R_{2} f\right)(s, t)=f\left(s, s^{-1} t\right)$.

This is a very simple example. And it can be combined with usual Hopf algebra examples to obtain more complicated ones. To give less straightforward examples is not so easy. In [5], we showed that multiplier Hopf algebras give a natural framework to study discrete quantum groups. Therefore, interesting examples of multiplier Hopf algebras are found among the discrete quantum groups (and so the duals of compact quantum groups).

\section{CONSTRUCTION OF THE COUNIT}

In this section we assume that $(A, \Delta)$ is a multiplier Hopf algebra. We will construct a homomorphism $\varepsilon: A \rightarrow \mathbb{C}$ that has the properties of a counit in usual Hopf algebra theory.

3.1 Definition. Define a map $E: A \rightarrow L(A)$, the left multiplier algebra of $A$, by

$$
E(a) b=m T_{1}^{-1}(a \otimes b)
$$

where $m$ denotes multiplication, considered as a linear map from $A \otimes A$ to $A$ and where $T_{1}$ is defined as before by $T_{1}(a \otimes b)=\Delta(a)(1 \otimes b)$. 
Because $T_{1}(x(1 \otimes c))=T_{1}(x)(1 \otimes c)$ for all $x$ in $A \otimes A$ and $c \in A$, the same result holds for $T_{1}^{-1}$. And since $m(x(1 \otimes c))=m(x) c$ we get that $E(a)$ is indeed a left multiplier on $A$ for all $a \in A$.

We will show that $E(A) \subseteq \mathbb{C} 1$ and this will give us the map $\varepsilon$. We need a lemma before we can do this.

3.2 Lemma. For all $a, b \in A$ we have

$$
(l \otimes E)((a \otimes 1) \Delta(b))=a b \otimes 1 .
$$

Proof. Assume $a, b \in A$ and let

$$
a \otimes b=\sum_{i=1}^{n} \Delta\left(a_{i}\right)\left(1 \otimes b_{i}\right) .
$$

If we apply $\Delta \otimes \imath$, multiply with $c \otimes 1 \otimes 1$ on the left and use coassociativity, we obtain

$$
\begin{aligned}
((c \otimes 1) \Delta(a)) \otimes b & =\sum(c \otimes 1 \otimes 1)(\Delta \otimes l)\left(\Delta\left(a_{i}\right)\left(1 \otimes b_{i}\right)\right) \\
& =\sum(l \otimes \Delta)\left((c \otimes 1) \Delta\left(a_{i}\right)\right)\left(1 \otimes 1 \otimes b_{i}\right) .
\end{aligned}
$$

Now let $\varphi$ be any linear functional on $A$ and apply $\varphi \otimes \imath \otimes l$ to the above equation. We get

$$
\begin{aligned}
(\varphi \otimes l)((c \otimes 1) \Delta(a)) \otimes b & =\sum \Delta\left((\varphi \otimes l)\left((c \otimes 1) \Delta\left(a_{i}\right)\right)\right)\left(1 \otimes b_{i}\right) \\
& =T_{1}\left(\sum(\varphi \otimes \imath)\left((c \otimes 1) \Delta\left(a_{i}\right)\right) \otimes b_{i}\right) .
\end{aligned}
$$

By the definition of $E$ we get

$$
E((\varphi \otimes \imath)((c \otimes 1) \Delta(a))) b=\sum(\varphi \otimes \imath)\left((c \otimes 1) \Delta\left(a_{i}\right)\right) b_{i} .
$$

So

$$
\begin{aligned}
(\varphi \otimes \imath)((l \otimes E)((c \otimes 1) \Delta(a))(1 \otimes b)) & =(\varphi \otimes \imath)\left((c \otimes 1) \sum \Delta\left(a_{i}\right)\left(1 \otimes b_{i}\right)\right) \\
& =(\varphi \otimes \imath)((c \otimes 1)(a \otimes b)) .
\end{aligned}
$$

Because this hold for all $\varphi$ we get

$$
(l \otimes E)((c \otimes 1) \Delta(a))(1 \otimes b)=(c a \otimes 1)(1 \otimes b) .
$$

This gives the required formula in the multiplier algebra.

3.3 Lemma. $E(A) \subseteq \mathbb{C} 1$.

Proof. By the surjectivity of the map $T_{2}$, defined by $T_{2}(a \otimes b)=(a \otimes 1) \Delta(b)$, we see that $a \otimes E(b) \in A \otimes 1$ for all $a$ and $b$ in $A$. This gives the result.

The we can define the counit.

3.4 Definition. Define $\varepsilon: A \rightarrow \mathbb{C}$ by $\varepsilon(a) 1=E(a)$.

We will show that $\varepsilon$ satisfies the usual properties of the counit in Hopf algebra theory. 
3.5 Lemma. $\varepsilon$ is a homomorphism.

Proof. By Lemma 3.2 we have

$$
(l \otimes \varepsilon)((a \otimes 1) \Delta(b c))=a b c
$$

for all $a, b, c \in A$. Then

$$
(l \otimes \varepsilon)((a \otimes 1) \Delta(b) \Delta(c))=(a b) c=(l \otimes \varepsilon)((a \otimes 1) \Delta(b)) c .
$$

By the surjectivity of $T_{2}$ we get

$$
\begin{aligned}
(l \otimes \varepsilon)((a \otimes b) \Delta(c)) & =(l \otimes \varepsilon)(a \otimes b) c=a \varepsilon(b) c=\varepsilon(b) a c \\
& =\varepsilon(b)(l \otimes \varepsilon)((a \otimes 1) \Delta(c))
\end{aligned}
$$

for all $a, b, c \in A$. Again by the surjectivity of $T_{2}$ we get

$$
(l \otimes \varepsilon)(a \otimes b c)=\varepsilon(b)(l \otimes \varepsilon)(a \otimes c) .
$$

This means

$$
a \varepsilon(b c)=a \varepsilon(b) \varepsilon(c) .
$$

Remark that we have used the bijectivity of $T_{1}$ to define $E$ and the surjectivity of $T_{2}$ to obtain that $E(A) \subseteq \mathbb{C} 1$ and that $E$ is a homomorphism. If, e.g., $A$ is an algebra with 1 and if $\Delta: A \rightarrow A \otimes A$ is defined by $\Delta(a)=a \otimes 1$, then $\Delta$ is a comultiplication such that $T_{1}=\imath$. Then $E$ would be defined and $E(a)=a$ for all $a \in A$. Of course, here $T_{2}$ is not surjective anymore.

The formula in Lemma 3.2 can be rewritten as

$$
(l \otimes \varepsilon)((a \otimes 1) \Delta(b))=a b .
$$

By the definition of $\varepsilon$ we also get

$$
(\varepsilon \otimes \imath)(a \otimes b)=m T_{1}^{-1}(a \otimes b)
$$

and hence

$$
(\varepsilon \otimes l)(\Delta(a)(1 \otimes b))=a b .
$$

These formulas just mean

$$
(l \otimes \varepsilon) \Delta=(\varepsilon \otimes l) \Delta=l,
$$

where now $l \otimes \varepsilon$ and $\varepsilon \otimes l$ are the unique extensions to $M(A \otimes A)$.

So, all together we obtain the following result.

3.6 Theorem. Let $A$ be a multiplier Hopf algebra. Then there is a homomorphism $\varepsilon: A \rightarrow \mathbb{C}$ such that

$$
(l \otimes \varepsilon)((a \otimes 1) \Delta(b))=a b, \quad(\varepsilon \otimes \imath)(\Delta(a)(1 \otimes b))=a b
$$

for all $a, b$.

It is clear that the above formulas determine $\varepsilon$ because of the surjectivity of the maps $T_{1}$ and $T_{2}$. If $A$ has an identity, then $\varepsilon$ is a counit in the usual sense. In $\S 5$, on regular multiplier Hopf algebras, we will also consider $*$-algebras. We will show that $\varepsilon$ is a $*$-homomorphism.

Let us finish this section by looking at our example. We see that, when $f, g \in A$ and $t \in G$,

$\varepsilon(f) g(t)=\left(m T_{1}^{-1}(f \otimes g)\right)(t)=\left(T_{1}^{-1}(f \otimes g)\right)(t, t)=(f \otimes g)\left(t t^{-1}, t\right)=f(e) g(t)$.

Hence (of course) $\varepsilon(f)=f(e)$. 


\section{Construction OF THE ANTIPODE}

In this section we will construct an antihomomorphism $S: A \rightarrow M(A)$ that has the properties of the antipode in the usual Hopf algebra theory.

4.1 Definition. Define a map $S: A \rightarrow L(A)$ by

$$
S(a) b=(\varepsilon \otimes \imath) T_{1}^{-1}(a \otimes b) .
$$

As before, also here $S(a)$ is indeed a left multiplier for all $a \in A$.

\subsection{Lemma.}

$$
(l \otimes S)((c \otimes 1) \Delta(a))(1 \otimes b)=(c \otimes 1) T_{1}^{-1}(a \otimes b) .
$$

Proof. As in the proof of Lemma 3.2, we have that, for $\varphi \in A^{\prime}$ and $a, b, c \in$ $A$,

$$
(\varphi \otimes \imath)((c \otimes 1) \Delta(a)) \otimes b=T_{1}\left(\sum(\varphi \otimes \imath)\left((c \otimes 1) \Delta\left(a_{i}\right)\right) \otimes b_{i}\right)
$$

if $a \otimes b=\sum \Delta\left(a_{i}\right)\left(1 \otimes b_{i}\right)$. Then, by the definition of $S$ we get

$$
\begin{aligned}
S((\varphi \otimes \imath)((c \otimes 1) \Delta(a))) b & =(\varepsilon \otimes l)\left(\sum(\varphi \otimes \imath)\left((c \otimes 1) \Delta\left(a_{i}\right)\right) \otimes b_{i}\right) \\
& =(\varphi \otimes \imath)\left(\sum(l \otimes \varepsilon)\left((c \otimes 1) \Delta\left(a_{i}\right)\right) \otimes b_{i}\right) \\
& =(\varphi \otimes \imath)\left(\sum c a_{i} \otimes b_{i}\right)=(\varphi \otimes \imath)\left((c \otimes 1) T_{1}^{-1}(a \otimes b)\right) .
\end{aligned}
$$

Hence

$$
(\varphi \otimes l)((l \otimes S)((c \otimes 1) \Delta(a))(1 \otimes b))=(\varphi \otimes l)\left((c \otimes 1) T_{1}^{-1}(a \otimes b)\right) .
$$

This is true for all $\varphi \in A^{\prime}$ and hence proves the result.

4.3 Lemma. For all $a, b, c \in A$ we have

$$
m((l \otimes S)((c \otimes 1) \Delta(a))(1 \otimes b))=c \varepsilon(a) b .
$$

Proof. We get this formula if we apply $m$ on the equation in Lemma 4.2 because

$$
\dot{m}\left((c \otimes 1) T_{1}^{-1}(a \otimes b)\right)=c m T_{1}^{-1}(a \otimes b)=c \varepsilon(a) b .
$$

4.4 Lemma. $S(a b)=S(b) S(a)$ for all $a, b \in A$.

Proof. We have

$$
\begin{aligned}
m((\imath \otimes S)((c \otimes 1) \Delta(a) \Delta(b))(1 \otimes d)) & =c \varepsilon(a b) d=c \varepsilon(a) d \varepsilon(b) \\
& =m((l \otimes S)((c \otimes 1) \Delta(a))(1 \otimes d)) \varepsilon(b)
\end{aligned}
$$

for all $a, b, c, d \in A$. By the surjectivity of $T_{2}$ we get

$$
\begin{aligned}
m((l \otimes S)((c \otimes a) \Delta(b))(1 \otimes d)) & =m((l \otimes S)(c \otimes a)(1 \otimes d)) \varepsilon(b) \\
& =c S(a) d \varepsilon(b)=c \varepsilon(b) S(a) d \\
& =m((l \otimes S)((c \otimes 1) \Delta(b))(1 \otimes S(a) d))
\end{aligned}
$$

for all $a, b, c, d \in A$. Again by the surjectivity of $T_{2}$ we get

$$
m((l \otimes S)(c \otimes a b)(1 \otimes d))=m((l \otimes S)(c \otimes b)(1 \otimes S(a) d))
$$

or $c S(a b) d=c S(b) S(a) d$. 
4.5 Lemma. $S(a)$ is also a right multiplier for all $a \in A$ and we have

$$
m((c \otimes 1)(S \otimes l)(\Delta(a)(1 \otimes b)))=c \varepsilon(a) b .
$$

Proof. Define $S^{\prime}: A \rightarrow R(A)$, the right multiplier algebra, by

$$
a S^{\prime}(b)=(l \otimes \varepsilon) T_{2}^{-1}(a \otimes b) .
$$

Because $T_{2}((a \otimes 1) x)=(a \otimes 1) T_{2}(x)$ for all $a \in A$ and $x \in A \otimes A$ we get indeed a right multiplier. Completely similar as in Lemma 4.2 we get here that

$$
(c \otimes 1)\left(S^{\prime} \otimes l\right)(\Delta(a)(1 \otimes b))=\left(T_{2}^{-1}(c \otimes a)\right)(1 \otimes b) .
$$

And if we apply $m$ we get the formula in the statement of the lemma with $S^{\prime}$ instead of $S$ because

$$
m\left(T_{2}^{-1}(c \otimes a)\right)=c \varepsilon(a) .
$$

We now show that $S=S^{\prime}$. Indeed, we have, by definition,

$$
a S^{\prime}(b)=\sum a_{i} \varepsilon\left(b_{i}\right)
$$

if $a \otimes b=\sum\left(a_{i} \otimes 1\right) \Delta\left(b_{i}\right)$. If we apply $l \otimes S$ and multiply with $1 \otimes c$ we get

$$
a \otimes S(b) c=\sum(l \otimes S)\left(\left(a_{i} \otimes 1\right) \Delta\left(b_{i}\right)\right)(1 \otimes c) .
$$

And if we apply $m$ we obtain, using 4.3 that

$$
a S(b) c=\sum a_{i} \varepsilon\left(b_{i}\right) c=a S^{\prime}(b) c .
$$

This shows that $S(b)=S^{\prime}(b)$. This proves the lemma; the formula was already proven for $S^{\prime}$.

In the proof we have also shown that

$$
(c \otimes 1)(S \otimes \imath)(\Delta(a)(1 \otimes b))=\left(T_{2}^{-1}(c \otimes a)\right)(1 \otimes b)
$$

for all $a, b, c$ in $A$. This is similar to the formula in Lemma 4.2. We will need these two formulas in the next section. Remark also that these formulas give the counterpart of the result in Proposition 2.1.

The main result can again be summarized as follows.

4.6 Theorem. If $A$ is a multiplier Hopf algebra then there exists an antihomomorphism $S: A \rightarrow M(A)$ such that

$$
\begin{aligned}
& m((l \otimes S)((c \otimes 1) \Delta(a))(1 \otimes b))=c \varepsilon(a) b, \\
& m((c \otimes 1)(S \otimes l)(\Delta(a)(1 \otimes b)))=c \varepsilon(a) b
\end{aligned}
$$

for all $a, b, c \in A$.

It is not so hard to show that the above formulas determine $S$, just as in the case of the counit. If $A$ has an identity, then $S$ is an antihomomorphism of $A$ and we have the usual formulas

$$
m(l \otimes S) \Delta(a)=\varepsilon(a) 1, \quad m(S \otimes l) \Delta(a)=\varepsilon(a) 1 .
$$

If we combine this with the results on $\varepsilon$ we obtain 
4.7 Theorem. If $A$ is a multiplier Hopf algebra with an identity, then $A$ is a Hopf algebra.

In the next section we will also consider the case of a $*$-algebra. We will have that, in that case $S$ is a map from $A$ to $A$, and that $S\left(S(a)^{*}\right)^{*}=a$ for all $a \in A$.

Again we finish this section by looking at our example. We have, when $f, g \in A$ and $t \in G$,

$$
\begin{aligned}
(S(f) g)(t) & =\left((\varepsilon \otimes l) T_{1}^{-1}(f \otimes g)\right)(t)=T_{1}^{-1}(f \otimes g)(e, t) \\
& =(f \otimes g)\left(t^{-1}, t\right)=f\left(t^{-1}\right) g(t) .
\end{aligned}
$$

Hence $S(f)(t)=f\left(t^{-1}\right)$.

\section{Regular multiplier Hopf algebras}

In this section $(A, \Delta)$ will be a regular multiplier Hopf algebra. Denote by $\Delta^{\prime}$ the opposite comultiplication. Then $\left(A, \Delta^{\prime}\right)$ is again a multiplier Hopf algebra. Let $\varepsilon^{\prime}$ and $S^{\prime}$ be the associated counit and antipode.

5.1 Lemma $\varepsilon=\varepsilon^{\prime}$.

Proof. If we rewrite the first formula in Theorem 3.6 for $\varepsilon^{\prime}$ we find that

$$
\left(\varepsilon^{\prime} \otimes \imath\right)((1 \otimes a) \Delta(b))=a b .
$$

On the other hand,

$$
(\varepsilon \otimes \imath)((1 \otimes a) \Delta(b)) c=a(\varepsilon \otimes \imath)(\Delta(b)(1 \otimes c))=a b c .
$$

Therefore also

$$
(\varepsilon \otimes l)((1 \otimes a) \Delta(b))=a b .
$$

The surjectivity of the map $a \otimes b \rightarrow(1 \otimes a) \Delta(b)$ shows that $\varepsilon=\varepsilon^{\prime}$.

So in the case of a regular algebra we get

$$
\begin{aligned}
& (\varepsilon \otimes l)((1 \otimes a) \Delta(b))=a b, \quad(\varepsilon \otimes l)(\Delta(a)(1 \otimes b))=a b, \\
& (l \otimes \varepsilon)((a \otimes 1) \Delta(b))=a b, \quad(l \otimes \varepsilon)(\Delta(a)(b \otimes 1))=a b
\end{aligned}
$$

for all $a, b \in A$.

The situation with the antipode is more difficult.

5.2 Proposition. $S(A) \subseteq A$ and $S^{\prime}(A) \subseteq A$ and $S$ and $S^{\prime}$ are each other's inverses.

Proof. If $a \otimes b=\sum \Delta\left(a_{i}\right)\left(1 \otimes b_{i}\right)$ we have $S(a) b=\sum \varepsilon\left(a_{i}\right) b_{i}$ by definition. Then

$$
b \otimes a=\sum \Delta^{\prime}\left(a_{i}\right)\left(b_{i} \otimes 1\right)
$$

and

$$
b \otimes a c=\sum \Delta^{\prime}\left(a_{i}\right)\left(b_{i} \otimes c\right) .
$$

If we apply $S^{\prime} \otimes l$ and multiply with $d \otimes 1$ we get

$$
\begin{aligned}
d S^{\prime}(b) \otimes a c & =\sum_{i}(d \otimes 1)\left(S^{\prime} \otimes \imath\right)\left(\Delta^{\prime}\left(a_{i}\right)\left(b_{i} \otimes c\right)\right) \\
& =\sum_{i}(d \otimes 1)\left(S^{\prime}\left(b_{i}\right) \otimes 1\right)\left(S^{\prime} \otimes \imath\right)\left(\Delta^{\prime}\left(a_{i}\right)(1 \otimes c)\right) .
\end{aligned}
$$


If we now multiply and use Lemma 4.5 for $\Delta^{\prime}$ we obtain

$$
d S^{\prime}(b) a c=\sum_{i} d S^{\prime}\left(b_{i}\right) \varepsilon\left(a_{i}\right) c=d S^{\prime}\left(\sum \varepsilon\left(a_{i}\right) b_{i}\right) c=d S^{\prime}(S(a) b) c .
$$

This shows that

$$
S^{\prime}(b) a=S^{\prime}(S(a) b) .
$$

From Definition 4.1 we see that elements of the form $S(a) b$ span $A$. Then the above formula implies that $S^{\prime}(A) \subseteq A$. Similarly also $S(A) \subseteq A$ and the above formula gives

$$
S^{\prime}(b) a=S^{\prime}(b) S^{\prime}(S(a)) .
$$

If we multiply with $c$ to the left and use that also elements of the form $c S^{\prime}(b)$ span $A$, we get $a=S^{\prime}(S(a))$. Similarly also $S\left(S^{\prime}(a)\right)=a$.

If $A$ is cocommutative, i.e., if $\Delta=\Delta^{\prime}$, then it is clear that $A$ is automatically regular and that $S=S^{\prime}$ so that $S^{2}=1$. This is also true when $A$ is commutative.

5.3 Proposition. If $A$ is an abelian multiplier Hopf algebra, then $A$ is regular and $S^{2}=1$.

Proof. It is easy to see that regularity also here is automatic. We prove now that $S=S^{\prime}$. Consider the second formula of Theorem 4.6 for $\Delta^{\prime}$. We get, using that $A$ is abelian,

$$
\begin{aligned}
c \varepsilon(a) b & =m\left((c \otimes 1)\left(S^{\prime} \otimes l\right)\left(\Delta^{\prime}(a)(1 \otimes b)\right)\right) \\
& =m\left(\left(S^{\prime} \otimes \imath\right)\left((1 \otimes b) \Delta^{\prime}(a)\right)(c \otimes 1)\right) \\
& =m \sigma\left(\left(l \otimes S^{\prime}\right)((b \otimes 1) \Delta(a))(1 \otimes c)\right) \\
& =m\left(\left(l \otimes S^{\prime}\right)((b \otimes 1) \Delta(a))(1 \otimes c)\right) .
\end{aligned}
$$

On the other hand, using the first formula in 4.6 for $\Delta$ and the fact that $A$ is abelian, we get

$$
m((l \otimes S)((b \otimes 1) \Delta(a))(1 \otimes c))=b \varepsilon(a) c=c \varepsilon(a) b .
$$

These two expressions are the same, and if we replace $(b \otimes 1) \Delta(a)$ by $b \otimes a$ we get

$$
m\left(\left(l \otimes S^{\prime}\right)(b \otimes a)(1 \otimes c)\right)=m((l \otimes S)(b \otimes a)(1 \otimes c)),
$$

and hence $b S^{\prime}(a) c=b S(a) c$. This proves that $S=S^{\prime}$.

Now we will prove that the formula $\Delta(S(a))=\sigma(S \otimes S) \Delta(a)$, which is true for usual Hopf algebras, is still true for regular multiplier Hopf algebras under an appropriate form. We need two lemmas to prove this. The first one is a reformulation of the formulas in Lemma 4.2 and the remark after Lemma 4.5.

5.4 Lemma. Suppose that $a, b, a_{i}, b_{i}$ are elements of $A$. Then

$$
a \otimes S b=\sum \Delta\left(a_{i}\right)\left(1 \otimes b_{i}\right) \quad \text { iff } \quad(1 \otimes b) \Delta(a)=\sum a_{i} \otimes S^{-1} b_{i}
$$

and

$$
S b \otimes a=\sum\left(b_{i} \otimes 1\right) \Delta\left(a_{i}\right) \quad \text { iff } \quad \Delta(a)(b \otimes 1)=\sum S^{-1} b_{i} \otimes a_{i} .
$$

Proof. Assume that $a \otimes S b=\sum \Delta\left(a_{i}\right)\left(1 \otimes b_{i}\right)$. By Lemma 4.2 we have for all $c \in A$ that

$$
\sum c a_{i} \otimes b_{i}=(l \otimes S)((c \otimes 1) \Delta(a))(1 \otimes S b)=(l \otimes S)((c \otimes b) \Delta(a)) .
$$


If we apply $l \otimes S^{-1}$ and cancel $c$ we get the required formula in the first statement. Now assume that $S b \otimes a=\sum\left(b_{i} \otimes 1\right) \Delta\left(a_{i}\right)$. By the formula after Lemma 4.5 we get, for all $c \in A$,

$$
\sum b_{i} \otimes a_{i} c=(S b \otimes 1)(S \otimes l)(\Delta(a)(1 \otimes c))=(S \otimes l)(\Delta(a)(b \otimes c)) .
$$

If we apply $S^{-1} \otimes l$ and cancel $c$ we get the second formula.

5.5 Lemma. Suppose that $a, b, a_{i}, b_{i}$ are elements of $A$. Then the following are equivalent:

(i) $\Delta(a)(1 \otimes b)=\sum \Delta\left(a_{i}\right)\left(b_{i} \otimes 1\right)$,

(ii) $a \otimes S^{-1} b=\sum\left(a_{i} \otimes 1\right) \Delta\left(b_{i}\right)$,

(iii) $(1 \otimes a) \Delta(b)=\sum S b_{i} \otimes a_{i}$.

Proof. Assume that $\Delta(a)(1 \otimes b)=\sum \Delta\left(a_{i}\right)\left(b_{i} \otimes 1\right)$. Let $c \in A$ and write

$$
b_{i} \otimes S c=\sum_{k} \Delta\left(p_{i k}\right)\left(1 \otimes q_{i k}\right)
$$

for all $i$. Then

$$
\Delta(a)(1 \otimes b S c)=\sum_{i, k} \Delta\left(a_{i} p_{i k}\right)\left(1 \otimes q_{i k}\right)
$$

and by the injectivity of the map $T_{1}$ we get

$$
a \otimes b S c=\sum_{i, k} a_{i} p_{i k} \otimes q_{i k} .
$$

On the other hand, by Lemma 5.4 we get also

$$
(1 \otimes c) \Delta\left(b_{i}\right)=\sum_{k} p_{i k} \otimes S^{-1} q_{i k}
$$

Therefore

$$
a \otimes c S^{-1} b=\sum_{i, k} a_{i} p_{i k} \otimes S^{-1} q_{i k}=\sum_{i, k}\left(a_{i} \otimes c\right) \Delta\left(b_{i}\right) .
$$

If we cancel $c$ we get the second formula.

Now assume that $\Delta(a)(b \otimes 1)=\sum \Delta\left(a_{i}\right)\left(1 \otimes b_{i}\right)$. If we apply the first equivalence for $\Delta^{\prime}$ and reverse the order in the tensor product, we obtain $S b \otimes A=\sum\left(1 \otimes a_{i}\right) \Delta\left(b_{i}\right)$. But this is, up to a different summation, essentially the equivalence of (i) and (iii).

We now prove the announced result.

5.6 Proposition. For all $a, b \in A$ we get

Proof. Write

$$
(1 \otimes S b) \Delta(S a)=(S \otimes S)\left(\Delta^{\prime}(a)(1 \otimes b)\right) .
$$

By Lemma 5.5 we find that

$$
\Delta(S b)(1 \otimes S a)=\sum \Delta\left(a_{i}\right)\left(b_{i} \otimes 1\right) .
$$

$$
S b \otimes a=\sum\left(a_{i} \otimes 1\right) \Delta\left(b_{i}\right), \quad(1 \otimes S b) \Delta(S a)=\sum S b_{i} \otimes a_{i} .
$$

If we apply the second equivalence of Lemma 5.4 to the first formula above, we get

$$
\Delta(a)(b \otimes 1)=\sum S^{-1} a_{i} \otimes b_{i} .
$$


If we combine all this we get

$$
\begin{aligned}
(1 \otimes S b)(\Delta(S a)) & =(S \otimes S)\left(\sum b_{i} \otimes S^{-1} a_{i}\right) \\
& =(S \otimes S) \sigma(\Delta(a)(b \otimes 1))=(S \otimes S)\left(\Delta^{\prime}(a)(1 \otimes b)\right) .
\end{aligned}
$$

Let us now consider the case of a $*$-algebra. Also in this case regularity is automatic. Here we have the following results for the behaviour of the counit and the antipode with respect to the involution.

5.7 Proposition. If $A$ is a multiplier Hopf *-algebra, then $\varepsilon$ is a *-homomorphism.

Proof. Define $\bar{\varepsilon}(a)=\varepsilon\left(a^{*}\right)^{-}$. Then

$$
\begin{aligned}
(\bar{\varepsilon} \otimes \imath)((1 \otimes a) \Delta(b)) & =\left((\varepsilon \otimes l)\left(\Delta\left(b^{*}\right)\left(1 \otimes a^{*}\right)\right)\right)^{*}=\left(b^{*} a^{*}\right)^{*}=a b \\
& =(\varepsilon \otimes l)((1 \otimes a) \Delta(b)) .
\end{aligned}
$$

This gives $\bar{\varepsilon}=\varepsilon$.

5.8 Proposition. If $A$ is a multiplier Hopf *algebra, then $S(A) \subseteq A$ and $S\left(S(a)^{*}\right)^{*}=a$ for all $a$.

Proof. If $a \otimes b=\sum \Delta\left(a_{i}\right)\left(1 \otimes b_{i}\right)$ we have $S(a) b=\sum \varepsilon\left(a_{i}\right) b_{i}$. If we take adjoints and apply the flip we will get

$$
b^{*} \otimes a^{*}=\sum\left(b_{i}^{*} \otimes 1\right) \Delta^{\prime}\left(a_{i}^{*}\right) .
$$

We now apply $\left(l \otimes S^{\prime}\right)$ and multiply with $1 \otimes c^{*}$ on the right. Using Lemma 4.3 for $\Delta^{\prime}$ we find

$$
b^{*} S^{\prime}\left(a^{*}\right) c^{*}=\sum b_{i}^{*} \varepsilon\left(a_{i}^{*}\right) c^{*}
$$

Taking adjoints we find

$$
c S^{\prime}\left(a^{*}\right)^{*} b=c S(a) b .
$$

This gives $S^{\prime}\left(a^{*}\right)=S(a)^{*}$. And because $S^{\prime}$ is the inverse of $S$ we get the desired formula.

\section{The DUAL ALGeBRA}

If $A$ is a usual Hopf *-algebra, the space $A^{\prime}$ of all linear functionals on $A$ can be made into a $*$-algebra. This is no longer possible for multiplier algebras because $(f \otimes g)(\Delta(a))$ need not be defined for all $f, g \in A^{\prime}$. We have to restrict the dual space.

6.1 Definition. Let $A^{*}$ be the vector space of linear functionals on $A$ spanned by elements of the form $a \rightarrow f(b a c)$ where $b, c \in A$ and $f \in A^{\prime}$.

We show that $A^{*}$ is an algebra.

6.2 Proposition. We can define a product on $A^{*}$ by $(f g)(a)=(f \otimes g)(\Delta(a))$, making $A^{*}$ into an associative algebra.

Proof. Let us first show that this product is well defined. Assume $f(a)=$ $f^{\prime}(b a c)$ and $g(a)=g^{\prime}(d a e)$ with $a, b, c, d, e \in A$ and $f^{\prime}, g^{\prime} \in A^{\prime}$. Then 
and we can apply $f^{\prime} \otimes g^{\prime}$. We get

$$
\left(f^{\prime} \otimes g^{\prime}\right)((b \otimes d) \Delta(a)(c \otimes e))=f(x)
$$

where $x=\left(l \otimes g^{\prime}\right)((1 \otimes d) \Delta(a)(1 \otimes e))$ and $x \in A$. This shows that the above expression extends by linearity to all $f, g \in A^{*}$. The result is a linear functional on $A$. We denote it by $f g$.

Now we show that $f g \in A^{*}$ when $f, g \in A^{*}$. Again assume $f(a)=f^{\prime}(b a c)$ and $g(a)=g^{\prime}(d a e)$ so that

$$
(f g)(a)=\left(f^{\prime} \otimes g^{\prime}\right)((b \otimes d) \Delta(a)(c \otimes e))
$$

We know that elements of the form $(p \otimes 1) \Delta(q)$ span $A \otimes A$. If we write $b \otimes d$ as a linear combination of such elements we find that $f g$ is a linear combination of maps

$$
a \rightarrow\left(f^{\prime} \otimes g^{\prime}\right)((p \otimes 1) \Delta(q a)(c \otimes e)) .
$$

Similarly, we can replace $c \otimes e$ by $\Delta(r)(1 \otimes s)$ and we have that $f g$ is a linear combination of maps

$$
a \rightarrow\left(f^{\prime} \otimes g^{\prime}\right)((p \otimes 1) \Delta(q a r)(1 \otimes s)) .
$$

If we let

$$
h(a)=\left(f^{\prime} \otimes g^{\prime}\right)((p \otimes 1) \Delta(a)(1 \otimes s))
$$

we see that $f g$ is spanned by functions of the form $a \rightarrow h(q a r)$ with $h \in A^{\prime}$ and $q, r \in A$. Hence $f g \in A^{*}$.

We now prove that this product is associative. Let $f, g, h \in A^{*}$. Assume $f(a)=f^{\prime}(b a c), g(a)=g^{\prime}(d a e)$, and $h(a)=h^{\prime}(p a q)$. Then

$$
\begin{aligned}
((f g) h)(a) & =\left(f g \otimes h^{\prime}\right)((1 \otimes p) \Delta(a)(1 \otimes q)) \\
& =\left(f^{\prime} \otimes g^{\prime} \otimes h^{\prime}\right)((b \otimes d \otimes 1)(\Delta \otimes l)((1 \otimes p) \Delta(a)(1 \otimes q))(c \otimes e \otimes 1)) \\
& =\left(f^{\prime} \otimes g^{\prime} \otimes h^{\prime}\right)((b \otimes d \otimes p)(\Delta \otimes l)(\Delta(a)(1 \otimes q))(c \otimes e \otimes 1)) \\
& =\left(f^{\prime} \otimes g^{\prime} \otimes h^{\prime}\right)((1 \otimes d \otimes p)(l \otimes \Delta)((b \otimes 1) \Delta(a))(c \otimes e \otimes q)) \\
& =\left(f^{\prime} \otimes g^{\prime} \otimes h^{\prime}\right)((1 \otimes d \otimes p)(l \otimes \Delta)((b \otimes 1) \Delta(a)(c \otimes 1))(1 \otimes e \otimes q)) \\
& =\left(f^{\prime} \otimes g h\right)((b \otimes 1) \Delta(a)(c \otimes 1)) \\
& =(f(g h))(a) .
\end{aligned}
$$

We, of course, used to coassociativity of $\Delta$ here, as formulated in Definition 2.2 .

If $f(a)=f^{\prime}(b a c)$ and if $\varepsilon$ is the counit of $A$ we get

$\left(\varepsilon \otimes f^{\prime}\right)((1 \otimes b) \Delta(a)(1 \otimes c))=f^{\prime}(b(\varepsilon \otimes l)(\Delta(a)(1 \otimes c)))=f^{\prime}(b a c)=f(a)$.

This shows that $(\varepsilon \otimes f) \Delta(a)=f(a)$. Similarly $(f \otimes \varepsilon) \Delta(a)=f(a)$. So $\varepsilon$ corresponds to the identity in the multiplier algebra of $A^{*}$. $A^{\prime}$ :

If $A$ is a regular multiplier Hopf algebra we can define the adjoint of $S$ on

6.3 Definition. If $A$ is a regular multiplier Hopf algebra and $S$ is the antipode, define $(S f)(a)=f(S(a))$ for $f \in A^{\prime}$ and $a \in A$. Then $S f \in A^{\prime}$ for all $f \in A^{\prime}$. 
6.4 Lemma. $S f \in A^{*}$ if $f \in A^{*}$.

Proof. Let $f(a)=f^{\prime}(b a c)$ with $f^{\prime} \in A^{\prime}$ and $b, c \in A$. Choose $d \in A$ such that $\varepsilon(d)=1$. Then, using Lemma 4.3, we get

$$
\begin{aligned}
(S f)(a) & =f(S(a))=f^{\prime}(b S(a) c)=f^{\prime}(b \varepsilon(d) S(a) c) \\
& =f^{\prime}(m((l \otimes S)((b \otimes 1) \Delta(d))(1 \otimes S(a) c))) .
\end{aligned}
$$

Now we have $(b \otimes 1) \Delta(d) \in A \otimes A$. So $S f$ is a linear combination of functionals of the form

$$
a \rightarrow f^{\prime}(m((l \otimes S)(b \otimes d)(1 \otimes S(a) c)))=f^{\prime}(b S(d) S(a) c)=f^{\prime}(b S(a d) c) .
$$

A similar argument gives us combinations of functions $a \rightarrow f^{\prime}(b S(e a d) c)$. This proves the lemma.

Now we want to show that $S$ is an antihomomorphism on $A^{*}$. We will of course use Proposition 5.6.

6.5 Proposition. If $A$ is a regular multiplier Hopf algebra then $S$ is an antihomomorphism of $A^{*}$.

Proof. Let $f, g \in A^{*}$ and write $g(a)=g^{\prime}(S(b) a)$ for some $g^{\prime}$ and $b$. Then

$$
\begin{aligned}
(S(f g))(a) & =(f g)(S(a))=\left(f \otimes g^{\prime}\right)(1 \otimes S(b)) \Delta(S(a)) \\
& =\left(f \otimes g^{\prime}\right)\left((S \otimes S)\left(\Delta^{\prime}(a)(1 \otimes b)\right)\right) \\
& =\left(S g^{\prime} \otimes S f\right)(\Delta(a)(b \otimes 1)) .
\end{aligned}
$$

Of course

$$
\left(S g^{\prime}\right)(a b)=g^{\prime}(S(b) S(a))=g(S(a))=(S g)(a) .
$$

Hence $(S(f g))(a)=((S g)(S f))(a)$.

For a $*$-algebra $A$ we get that $A^{*}$ is a $*$-algebra.

6.6 Proposition. If we define $f^{*} \in A^{\prime}$ by $f^{*}(a)=f\left(S(a)^{*}\right)^{-}$then $f^{*} \in A^{*}$ when $f \in A^{*}$ and $A^{*}$ becomes a $*$-algebra.

Proof. If $f \in A^{\prime}$ define $\bar{f}$ by $\bar{f}(a)=f\left(a^{*}\right)^{-}$. If $f(a)=f^{\prime}(b a c)$ then $f\left(a^{*}\right)^{-}=f^{\prime}\left(b a^{*} c\right)^{-}=f^{\prime}\left(\left(c^{*} a b^{*}\right)^{*}\right)^{-}$. It follows that $\bar{f} \in A^{*}$. Now $f^{*}(a)=$ $\bar{f}(S(a))$. So also $f^{*} \in A^{*}$. Because $S\left(S(a)^{*}\right)^{*}=a$ for all $a$ we clearly have $f^{* *}=f$ for all $f$. Furthermore

$$
\begin{aligned}
\overline{(f g)}(a) & =(f g)\left(a^{*}\right)^{-}=(f \otimes g)\left(\Delta\left(a^{*}\right)\right)^{-}=(f \otimes g)\left(\Delta(a)^{*}\right)^{-} \\
& =(\bar{f} \otimes \bar{g})(\Delta(a))=(\bar{f} \cdot \bar{g})(a) .
\end{aligned}
$$

So $(f g)^{*}=S(\overline{f g})=S(\bar{f} \bar{g})=(S \bar{g})(S \bar{f})=g^{*} f^{*}$.

\section{ACKNOWLEDGMENT}

The results in this paper were obtained during our visit at the University of Trondheim. We are very grateful to $M$. Landstad and C. Skau, whose warm hospitality made our visit very enjoyable and mathematically fruitful. 


\section{APPENDIX. MULTIPLIERS}

Let $A$ be an algebra over $\mathbb{C}$, with or without identity.

A.1 Definition. A left multiplier of $A$ is a linear map $\rho: A \rightarrow A$ such that $\rho(a b)=\rho(a) b$ for all $a, b \in A$. A right multiplier of $A$ is a linear map $\rho: A \rightarrow A$ such that $\rho(a b)=a \rho(b)$ for all $a, b \in A$. A multiplier of $A$ is a pair $\left(\rho_{1}, \rho_{2}\right)$ of a left and a right multiplier such that $\rho_{2}(a) b=a \rho_{1}(b)$ for all $a, b \in A$.

We denote by $L(A), R(A)$, and $M(A)$ the left, right, and multipliers of $A$. It is clear that the composition of maps makes these vector spaces into algebras.

If the product in $A$ is nondegenerate, i.e., if $a b=0$ for all $b$ implies $a=0$ and $a b=0$ for all $a$ implies $b=0$, then it is clear that left and right multiplication give a natural imbedding from $A$ into $L(A), R(A)$, and $M(A)$. Of course, if $A$ has an identity, the product is automatically nondegenerate and $L(A)=R(A)=M(A)=A$. If the product is nondegenerate, we will write $a b$ for $a(b)$ when $a \in L(A), b \in A, a b$ for $b(a)$ when $b \in R(A), a \in A$, and $a b$ for $a_{1}(b)$ and $b a$ for $a_{2}(b)$ when $a=\left(a_{1}, a_{2}\right)$ is an element of $M(A)$ and $b \in A$.

If $A$ is a *-algebra, we define $a b^{*}=\left(b a^{*}\right)^{*}$ for $a \in A$ and a left multiplier $b$. It is clear that $b^{*}$ is a right multiplier. If $b$ is a multiplier, we use the same formula to define the adjoint $b^{*}$. It will again be a multiplier. It is straightforward to check that $M(A)$ is a *-algebra and that $A$ is a *-subalgebra.

Now, let $A$ and $B$ be two algebras, with a nondegenerate product. Consider the tensor product $A \otimes B$ with the usual tensor product algebra structure.

A.2 Lemma. The product in $A \otimes B$ is again nondegenerate.

Proof. Consider $x=\sum a_{i} \otimes b_{i}$ in $A \otimes B$ and assume that $(c \otimes d) x=0$ for all $c \in A$ and $d \in B$. Then, for every linear functional $\varphi \in B^{\prime}$ we get

$$
c \sum a_{i} \varphi\left(d b_{i}\right)=0
$$

for all $c$. Because the product in $A$ is nondegenerate we must have

$$
\sum a_{i} \varphi\left(d b_{i}\right)=0 \text {. }
$$

Since this is true for all $\varphi$ we have

$$
\sum a_{i} \otimes d b_{i}=0 .
$$

By a similar argument, using the nondegeneracy of the product in $B$, we get $x=0$. Similarly for right multiplication.

A.3 Proposition. We have natural imbeddings $L(A) \otimes L(B) \hookrightarrow L(A \otimes B)$, $R(A) \otimes R(B) \hookrightarrow R(A \otimes B), M(A) \otimes M(B) \hookrightarrow M(A \otimes B)$.

Proof. We show this for the left multipliers. If $c \in L(A)$ and $d \in L(B)$ we define

$$
\varphi(c \otimes d)(a \otimes b)=c a \otimes d b .
$$

It is clear that $\varphi(c \otimes d)$ gives a left multiplier and that $\varphi$ extends to a linear map from $L(A) \otimes L(B)$ to $L(a \otimes B)$. The injectivity of $\varphi$ is essentially proved by the same technique as in Lemma A.2.

Consider a homomorphism $\varphi: A \rightarrow M(B)$. 
A.4 Definition. We call $\varphi$ nondegenerate if $B$ is spanned by vectors $\varphi(a) b$ and spanned by vectors $b \varphi(a)$.

Such homomorphisms have unique extensions.

A.5 Proposition. If $\varphi$ is a nondegenerate homomorphism from $A$ to $M(B)$, then $\varphi$ has a unique extension to a homomorphism $M(A) \rightarrow M(B)$.

Proof. If $\varphi_{1}$ is such an extension, we must have

$$
\varphi(c a) b=\varphi_{1}(c) \varphi(a) b
$$

when $c \in M(A), a \in A$, and $b \in B$. Because the elements $\varphi(a) b$ span $B$, we get uniqueness of $\varphi_{1}$.

Now we want to define $\varphi_{1}$ by the above formula. We first have to show that

$$
\sum \varphi\left(a_{i}\right) b_{i}=0 \Rightarrow \sum \varphi\left(c a_{i}\right) b_{i}=0
$$

for all $c \in M(A)$. So suppose $\sum \varphi\left(a_{i}\right) b_{i}=0$. Then for all $c \in M(A)$ and $d \in A, e \in B$ we get

$$
e \varphi(d) \sum \varphi\left(c a_{i}\right) b_{i}=e \sum \varphi\left(d c a_{i}\right) b_{i}=e \varphi(d c) \sum \varphi\left(a_{i}\right) b_{i}=0 .
$$

Because also $e \varphi(d)$ span $B$ we get

$$
\sum \varphi\left(c a_{i}\right) b_{i}=0 \text {. }
$$

Then we can define $\varphi_{1}$ on $M(A)$ by

$$
\varphi_{1}(c) \varphi(a) b=\varphi(c a) b .
$$

It is easy to show that $\varphi_{1}$ is still a homomorphism, and that it extends $\varphi$.

We consider the situation of $*$-algebras.

A.6 Proposition. If $\varphi$ is a nondegenerate $*$-homomorphism from $A \rightarrow M(B)$. Then it extends uniquely to a $*$-homomorphism from $M(A)$ to $M(B)$.

Proof. Let $\varphi_{1}$ be the unique extension to a homomorphism. We have

$$
\varphi(c a) b=\varphi_{1}(c) \varphi(a) b
$$

when $c \in M(A)$ and $a \in A$ and $b \in B$. Take adjoints and multiply with $\varphi\left(a_{1}^{*}\right) b_{1}^{*}$ where $a_{1} \in A, b_{1} \in B$ to obtain

$$
\begin{aligned}
b^{*} \varphi\left(a^{*}\right) \varphi_{1}(c)^{*} \varphi\left(a_{1}^{*}\right) b_{1}^{*} & =b^{*} \varphi\left(a^{*} c^{*}\right) \varphi\left(a_{1}^{*}\right) b_{1}^{*}=b^{*} \varphi\left(a^{*} c^{*} a_{1}^{*}\right) b_{1}^{*} \\
& =b^{*} \varphi\left(a^{*}\right) \varphi_{1}\left(c^{*}\right) \varphi\left(a_{1}^{*}\right) b_{1}^{*} .
\end{aligned}
$$

This implies that $\varphi_{1}(c)^{*}=\varphi_{1}\left(c^{*}\right)$.

\section{REFERENCES}

1. E. Abe, Hopf algebras, Cambridge Univ. Press, London and New York, 1977.

2. S. Baaj and G. Skandalis, Unitaires multiplicatifs et dualité pour les produits croisés de $C^{*}$-algèbres, Ann. Sci. Ecole Norm. Sup (4) 26 (1993), 425-488.

3. M. E. Sweedler, Hopf algebras, Math. Lecture Notes Ser., Benjamin, New York, 1969.

4. A. Van Daele, Dual pairs of Hopf *-algebras, Bull. London Math. Soc. 25 (1993), 209-230.

5. __ Discrete quantum groups, preprint K. U. Leuven (August 1993).

6. S. L. Woronowicz, Compact matrix pseudogroups, Comm. Math. Phys. 111 (1987), 613-665.

Department of Mathematics, K. U. Leuven, Celestijnenlaan 200B, B-3001 Heverlee, BELGIUM

E-mail address: fgaef02@cc3.kuleuven.ac.be 